\title{
Extending the potential of moment analysis in chromatography
}

\author{
Shamsul Qamar ${ }^{\mathrm{a}, \mathrm{b}, *}$, Andreas Seidel-Morgenstern ${ }^{\mathrm{a}}$ \\ ${ }^{a}$ Max Planck Institute for Dynamics of Complex Technical Systems Magdeburg, Germany \\ ${ }^{b}$ Department of Mathematics, COMSATS Institute of Information Technology Islamabad, Pakistan
}

\begin{abstract}
Essential goal of modeling chromatographic processes is to describe the dynamics of concentration fronts traveling through chromatographic columns. Most of the models developed originate from differential mass balances for the fluid and solid phases. Model reduction based on evaluating just a limited number of moments of the profiles is known to be a powerful tool to simplify the description of band profiles. This review article first describes the well-established method of moments for different standard models. Then the method is extended to evaluate more complex and realistic column models. The cases of applying columns packed with core-shell particles and the quantitative description of radial concentration profiles are analyzed.
\end{abstract}

Key words: Dynamics of chromatography, peak shapes, method of moments, Laplace transformation, finite volume scheme.

\section{Introduction}

Most of the mathematical models for chromatographic columns originate from differential mass balances for the fluid and solid phases [1-4]. Information about the column effluent profiles is the key for a rational process design and optimization. However, often the original differential mass balances models require the application of time consuming numerical solution techniques and it is often sufficient to condense the information of the complete concentration profile into a few characteristic features. Moment analysis (MA) is well-

\footnotetext{
${ }^{*}$ Corresponding author. Tel: +49-391-6110454; fax: +49-391-6110500

Email addresses: qamar@mpi-magdeburg.mpg.de (Shamsul Qamar)
} 
established method that provides condense information in the form of relatively small number of temporal moments. It can be applied a) to describe in a simpler manner essential feature of the chromatograms, b) to estimate efficiently free model parameters by matching measured and predicted moments, c) to predict performance parameters of the separations and, thus, d) to optimize more easily the process [2, 5-10]. In this study, we address essentially just the aspect a). Regarding the importance of these moments there is a clear hierarchy about the quality of representing chromatograms. It is more crucial that there is an agreement between predicted and measured values for lower order moments than for higher order moments. This is due to the fact that the zeroth moment describes the sample mass or peak area, the first moment corresponds to the mean retention time, the second moment quantifies the peak width or column efficiency, and the third moment represents the peak asymmetry (skewness). The fourth order moment still has a physical meaning (kurtosis) but is already difficult to measure precisely and because of that reason is hardly evaluated. Whereas there is a clear integration formula available to determine the moments from experimentally observed profiles, there is no simple connection between the thermodynamic and kinetic parameters of the chromatographic models, the operating parameters of the process and the corresponding theoretical moments. For the simplified situation of linear distribution equilibria for many of the chromatography models the moment generating property of the Laplace transform can be efficiently used to derive analytical expressions for the temporal moments. The method develops a new strategy for the analysis of chromatographic behavior beyond the ordinary plate and rate theories of chromatography [5-10].

Moment analysis has been comprehensively elaborated in the chromatographic literature [2, 7-20]. In these partly classical papers analytical moments were derived for specific chromatographic models and boundary conditions. The analysis typically covered just the most important first and second moments, i.e. retention times and band broadening. Beside the first and second moments, in a few studies also the third moment, which describes the peak asymmetry, was derived and evaluated, e.g. in the work of Prof. G. Guiochon [5, 6]. In our recently published couple of papers, we addressed several aspects that have not been 
considered to this depth up to now [21-25]. Apart from considering the three standard chromatographic models, we derived and compared also the fourth order moment, i.e. the kurtosis or flatness of the profile. Using low-noise detectors and complete capture of the responses this moment appears to be still experimentally accessible. As discussion on the influence of the boundary conditions (BCs) were often ignored in the literature, we compared the moment analysis for Dirichlet and Danckwerts BCs considering both rectangular pulses and steps as inlet profiles. For quantitative comparison, the first four moments of the General Rate Model (GRM), the Lumped Kinetic Model (LKM), and the Equilibrium Dispersive Model (EDM) were derived. With this analysis it was intended to elucidate the connections between the specific kinetic parameters, including for the first time the results for the fourth moments. Finally, going beyond previous studies, we provided a comparisons of the analytically derived moments with moments calculated independently by integrating numerically calculated effluent profiles. For this purpose advanced high resolution finite volume scheme was applied, which is capable to treat also the more general case of nonlinear equilibria [26]. We have recently extended the aforementioned analysis to core-shell particles using the GRM [25]. Core-shell particles were invented and pioneered by Horvath et al. [27] with the specific purpose of preparing columns that could provide highly efficient HPLC separation of high molecular weight compounds of biological origin. They are beneficial over fully porous beads in reducing diffusional mass transfer resistances in particle macropores and separation times. Furthermore, they can be also useful to regulate bead densities. Several researchers, including Guiochon and his co-authors, worked on the understanding and improvement of coreshell particles performance [28-35]. The analysis of one-dimensional (1D) models was recently extended to the analysis of two-dimensional (2D) models describing the movement of a solute in a two-dimensional chromatographic column of radial geometry [24]. In this case, the finite Hankel and Laplace transformations were simultaneously applied to solve the model equations. After eliminating the radial coordinate by Hankel transformation, the Laplace transformation was applied to solve the model equations analogously to the solution of 1D models.

In this review article, we provide a summary of the above mentioned instructive solutions 
by focusing on the: a) derivation of analytical expressions of first four temporal moments for the GRM and LKM using fully porous particles, b) derivation of first three temporal moments of GRM for core-shell particles, and on the c) derivation of first four temporal moments for the 2D GRM. To derive analytical moment expressions for the latter case, the Hankel transformation needs to be applied initially. The analysis is mainly focused on the derivation of temporal moments for the general rate model (GRM), while the temporal moments of LKM are derived as limiting cases of the GRM moments. The moments of simplified Equilibrium Dispersive Model (EDM) can be deduced from the moments of LKM by considering fast transport.

The structure of the article is as follows. In Section 2, the 1D GRM, 1D LKM and 2D GRM are briefly introduced. Section 3 presents the moments of 1D GRM and LKM for fully porous particles using Dirichlet BC. Section 4 presents the temporal moments of the 1D GRM for core-shell particles considering both Dirichlet and Danckwerts BCs. The first four temporal moments of 2D GRM are presented in Section 5 for Dirichlet BC. A few selected numerical test problems are presented in Section 6 for illustrating the results. Concluding remarks are given in Section 7.

\section{Mathematical models}

This section briefly introduces the two standard models of liquid chromatography considering fully porous stationary particles, namely the GRM and LKM. These mathematical models are typically derived using the following general assumptions $[1,2,4]$ :

1. The chromatographic process is isothermal.

2. The bed is homogeneous and the packing material used in the stationary phase is made of porous spherical particles of uniform size.

3. Axial dispersion occurs and causes band broadening.

4. The mobile phase is considered to be incompressible which holds for liquid chromatography. 
5. There is no interaction between the mobile and stationary phases.

In the $1 \mathrm{D}$ models the radial concentration gradients in the column are neglected, while in the 2D models such gradients are also considered.

\subsection{The 1D General Rate Model (GRM)}

The GRM considers several contributions of mass transfer kinetics occurring in chromatography $[1,2,4,18]$. As there are several ways to describe these effects, there are many versions of this model. Usually, axial dispersion, mass transfer between mobile and stationary phases and intraparticle, and pore diffusion are included in the equations. However, the possible limited rates of adsorption-desorption are often still ignored. The GRM contains two mass balances for the solute, one for inside the particles, and the other for outside the particles. The corresponding mass balance for a single-solute fluid percolating through a bed of spherical particles of radius $R_{P}$ is given as $[1,2,4]$

$$
\frac{\partial c}{\partial t}+u \frac{\partial c}{\partial z}=D_{L} \frac{\partial^{2} c}{\partial z^{2}}-\frac{3}{R_{p}} F k_{\mathrm{ext}}\left(c-\left.c_{p}\right|_{r=R_{p}}\right) .
$$

In the above equation, $c$ and $c_{p}$ are the concentrations of the solute in the bulk of the fluid and in particle pores, respectively. The phase ratio $F=(1-\epsilon) / \epsilon$, where $\epsilon \in(0,1)$ is the external porosity. Moreover, $u$ is the interstitial velocity, $D_{L}$ represents the axial dispersion, $k_{\text {ext }}$ is the external mass transfer coefficient, $R_{p}$ is the radius of stationary phase particle, and $t$ and $z$ denote time and axial coordinate of the column. In addition, $r$ denotes the radial coordinate along the particle radius.

The mass balance inside the particles is given as $[1,2,4]$

$$
\epsilon_{p} \frac{\partial c_{p}}{\partial t}+\left(1-\epsilon_{p}\right) \frac{\partial q_{p}^{*}}{\partial t}=\frac{1}{r^{2}} \frac{\partial}{\partial r}\left(r^{2}\left[\epsilon_{p} D_{p} \frac{\partial c_{p}}{\partial r}+\left(1-\epsilon_{p}\right) D_{s} \frac{\partial q_{p}^{*}}{\partial r}\right]\right)
$$

where $q_{p}^{*}$ is the local concentration of solute in the stationary phase, $\epsilon_{p}$ is the internal porosity, $D_{p}$ is the pore diffusivity, and $D_{s}$ is the surface diffusivity.

In this model it is assumed that kinetics of adsorption-desorption on the stationary phase are fast and their contribution to band broadening is negligible compared to the other contributions $[5,6]$. 
Only linear adsorption isotherms are considered in this work $[1,2]$ :

$$
q_{p}^{*}=a c_{p}
$$

By using Eq. (3), Eq. (2) can be simplified as

$$
a^{*} \frac{\partial c_{p}}{\partial t}=\frac{D_{\mathrm{eff}}}{r^{2}} \frac{\partial}{\partial r}\left(r^{2} \frac{\partial c_{p}}{\partial r}\right),
$$

where

$$
a^{*}=\epsilon_{p}+\left(1-\epsilon_{p}\right) a, \quad D_{\text {eff }}=\epsilon_{p} D_{p}+\left(1-\epsilon_{p}\right) D_{s} a .
$$

The Eqs. (1) and (4) are also subjected to the initial and boundary conditions. The initial conditions for an initially regenerated column are given as

$$
c(0, z)=0, \quad c_{p}(0, z, r)=0, \quad \forall z \in(0, L), r \in\left(0, R_{p}\right) .
$$

Here, $L$ represents the length of the column. Appropriate inlet and outlet boundary conditions (BCs) are required for Eq. (1). The following two types of boundary conditions are considered.

\section{Boundary conditions of type I: Dirichlet inlet BCs}

In this case, the simpler Dirichlet boundary conditions could be applied at the column inlet $[1,2,18]$ :

$$
\left.c\right|_{z=0}=\left\{\begin{array}{lr}
c_{\mathrm{inj}}, & \text { if } 0<t \leq t_{\mathrm{inj}}, \\
0, & t>t_{\mathrm{inj}}
\end{array}\right.
$$

together with a Neumann boundary condition for a column of hypothetically infinite length, $z=\infty:$

$$
\frac{\partial c(t, \infty)}{\partial z}=0 .
$$

For sufficiently small dispersion coefficient, this Dirichlet inlet boundary condition is well applicable. 


\section{Boundary conditions of type II: Danckwerts (or Robin) type inlet BCs}

Alternatively, the Robin type boundary condition, known in chemical engineering as Danckwerts boundary condition, could be applied at the column inlet $[2,36]$ :

$$
-\left.\frac{D_{L}}{u} \frac{\partial c}{\partial z}\right|_{z=0}+\left.c\right|_{z=0}=\left\{\begin{array}{lr}
c_{\mathrm{inj}}, & \text { if } 0<t \leq t_{\mathrm{inj}} \\
0, & t>t_{\mathrm{inj}}
\end{array}\right.
$$

where $c_{\text {inj }}$ denotes the injected concentration and $t_{\text {inj }}$ is the time of injection. At the outlet of the column of finite length $z=L$, the following Neumann outflow BCs are used:

$$
\frac{\partial c(t, L)}{\partial z}=0
$$

For sufficiently small dispersion coefficient Eq. (8a) reduces to Eq. (7a). For Eq. (4), the following boundary conditions at $r=0$ and $r=R_{p}$ are assumed [1,2]:

$$
\left.\frac{\partial c_{p}}{\partial r}\right|_{r=0}=0,\left.\quad D_{\text {eff }} \frac{\partial c_{p}}{\partial r}\right|_{r=R_{p}}=k_{\text {ext }}\left(c-\left.c_{p}\right|_{r=R_{p}}\right) .
$$

\subsection{The 1D Lumped Kinetic Model (LKM)}

The LKM incorporates the rate of variation of the local concentration of solute in the stationary phase and a local deviation from equilibrium concentrations. The model lumps the contribution of internal and external mass transport resistances into a mass transfer coefficient $k_{\text {LKM }}$. The model contains two mass balances, one for the liquid phase and one for the solid phase. For the liquid phase it can be expressed as $[1,2,18]$

$$
\frac{\partial c}{\partial t}+u \frac{\partial c}{\partial z}=D_{L} \frac{\partial^{2} c}{\partial z^{2}}-\frac{k_{\mathrm{LKM}}}{\epsilon}\left[q^{*}-q\right] .
$$

For the solid phase it is written as $[1,2,18]$

$$
\frac{\partial q}{\partial t}=\frac{k_{\mathrm{LKM}}}{(1-\epsilon)}\left[q^{*}-q\right] .
$$

The two conservation Eqs. (10) and (11) represent a typical 1D heterogeneous two-phase flow model. The same initial and boundary conditions can be used as given by Eqs. (6)(8b). 
If instead of $D_{L}$ and $k_{\text {LKM }}$, an apparent (lumped) dispersion coefficient $D_{\text {app }}$ is used $\left(D_{\text {app }}\left(D_{L}, k_{\mathrm{LKM}}\right)>D_{L}\right)$, then Eqs. (10) and (11) can be lumped together to obtain the well-known Equilibrium Dispersive Model (EDM) which is well studied and not considered here $[2]$.

\subsection{The 2D GRM}

The 2D GRM describes the movement of a solute in a two-dimensional chromatographic column of radial geometry as shown graphically in Figure 1. The injected solute moves in the $z$-direction by advection and axial dispersion, while it spreads in the $\rho$-direction by radial dispersion. To trigger and amplify the effect of possible rate limitations of the mass transfer in the radial direction, the following particular injection conditions are assumed. By introducing a parameter $\tilde{\rho}$, the inlet cross-section of the column is partitioned into an inner cylindrical core and an outer annular ring (see Figure 1). The injection profile is formulated in a general way allowing for injection either through an inner core, an outer ring or through the whole cross section. The latter case results if $\tilde{\rho}$ is set equal to the radius of the column denoted by $R$. Since in the latter case no initial radial gradients are provided, the solutions should converge into the solution of the simpler one-dimensional model.

As both axial and radial dispersion are considered, the 1D mass balance equation in Eq. (1) is replaced by $[2,23]$ :

$$
\frac{\partial c}{\partial t}+u \frac{\partial c}{\partial z}=D_{L} \frac{\partial^{2} c}{\partial z^{2}}-\frac{3}{R_{p}} F k_{\mathrm{ext}}\left(c-c_{p}\left(r=R_{p}\right)\right)+D_{\rho}\left(\frac{\partial^{2} c}{\partial \rho^{2}}+\frac{1}{\rho} \frac{\partial c}{\partial \rho}\right) .
$$

In the above equation, $D_{\rho}$ represents the radial dispersion coefficient and $\rho$ is the cylindrical coordinate of the column of radius $R$.

However, the structure of mass balance inside the particles (Eq. (2) or Eq. (4)), initial conditions (Eq. (6)), and boundary conditions (Eq. (9)) remain unchanged and are the integral parts of the 2D GRM.

For the current 2D model, the Danckwerts BCs in Eqs. (8a) and (8b) are replaced by the 
following BCs. For injection in the inner circular region, the inlet BC is expressed as [23]

$$
-\frac{D_{L}}{u} \frac{\partial c}{\partial z}+\left.c\right|_{z=0}= \begin{cases}c_{\mathrm{inj}}, & \text { if } 0 \leq \rho \leq \tilde{\rho} \text { and } 0 \leq t \leq t_{\mathrm{inj}}, \\ 0, & \text { if } \tilde{\rho}<\rho \leq R \text { or } t>t_{\mathrm{inj}},\end{cases}
$$

while, for injection in the outer annular zone, we have

$$
-\frac{D_{L}}{u} \frac{\partial c}{\partial z}+\left.c\right|_{z=0}= \begin{cases}c_{\mathrm{inj}}, & \text { if } \tilde{\rho} \leq \rho \leq R \text { and } 0 \leq t \leq t_{\mathrm{inj}} \\ 0, & \text { if } 1<\rho \leq \tilde{\rho} \text { or } t>t_{\mathrm{inj}} .\end{cases}
$$

At the outlet of the column of finite length $L$, the same Neumann outflow BC is used, i.e.

$$
\frac{\partial c_{i}(\rho, L, t)}{\partial z}=0 .
$$

When the dispersion coefficient $D_{L}$ is small, the boundary conditions reduce to the Dirichlet boundary conditions by neglecting the first terms on the left hand sides of Eqs. (13) and (14) and putting $L=\infty$ in Eq. (15).

\section{1D Moment equations for fully porous particles}

Moment analysis is an effective strategy for extracting condensed information about the retention and mass transfer kinetics in a chromatographic column [2, 5-20]. The moment generating property of the Laplace domain solutions can be utilized to obtain moments [5, 6, 21-23]. The retention equilibrium-constant and parameters of the mass transfer kinetics in the column are related to the moments of the Laplace domain solutions. In this section, the temporal moments up to fourth order are derived for the 1D GRM considering the fully porous particles, the Dirichlet boundary conditions (BCs) (c.f. Eqs. (7a) and (7b)), and rectangular pulse injections (finite feed volumes) [23, 25]. The moments of $1 \mathrm{D}$ LKM and EDM are derived from those of GRM as limiting cases [21, 22].

In order to calculate analytical temporal moments, the moment generating property of the Laplace transform is exploited [38]

$$
\mu_{n}=(-1)^{n} \frac{1}{\mu_{0}} \lim _{s \rightarrow 0} \frac{d^{n}(\bar{c}(s, z=L))}{d s^{n}}, \quad n=1,2,3, \cdots .
$$


The zeroth moment $\mu_{0}$ is expressed as

$$
\mu_{0}=\lim _{s \rightarrow 0} \bar{c}(s, z=L)
$$

The first three central moments are deduced from the normalized temporal moments $\mu_{n}$ using the expressions $[21,22]$

$$
\begin{aligned}
& \mu_{2}^{\prime}=\mu_{2}-\mu_{1}^{2}, \\
& \mu_{3}^{\prime}=\mu_{3}-3 \mu_{1} \mu_{2}+2 \mu_{1}^{3}, \\
& \mu_{4}^{\prime}=\mu_{4}-4 \mu_{1} \mu_{3}+6 \mu_{1}^{2} \mu_{2}-3 \mu_{1}^{4} .
\end{aligned}
$$

By applying the above definitions on the Laplace domain solutions, we obtain the following moments $[5,23]$

Zeroth moment: For a rectangular profile it is expressed as [5, 6, 23]

$$
\mu_{0, \mathrm{GRM}}=c_{\mathrm{inj}} t_{\mathrm{inj}}
$$

Here, $c_{\text {inj }}$ represents the concentration of the injected pulse to the column and $t_{\text {inj }}$ is the total time of injection. For continuous breakthrough curve it simplifies to

$$
\mu_{0, \mathrm{GRM}}=c_{\mathrm{inj}}
$$

The zeroth moment corresponds to the mass of the elution peak. This moment is the same for all three models, i.e. $\mu_{0, \mathrm{GRM}}=\mu_{0, \mathrm{LKM}}=\mu_{0, \mathrm{EDM}}$.

\section{First moment:}

The first temporal moment for rectangular profiles is given as $[5,6,23]$

$$
\mu_{1, \mathrm{GRM}}=\frac{t_{\mathrm{inj}}}{2}+\frac{L}{u}\left(1+a^{*} F\right), \quad a^{*}=\epsilon_{p}+\left(1-\epsilon_{p}\right) a
$$

The first moment $\mu_{1}$ corresponds to the retention time $t_{R}$. The value of the equilibrium constant $a$ can be estimated from the slopes of a straight lines, $\mu_{1}=t_{R}$ over $1 / u$ for constant column length and porosity. Eq. (23) reduces to the first moments of LKM by putting $\epsilon_{p}=0$, i.e. $a^{*}=a$. Thus, we obtain

$$
\mu_{1, \mathrm{LKM}}=\frac{t_{\mathrm{inj}}}{2}+\frac{L}{u}(1+a F) .
$$


Moreover, $\mu_{1, \mathrm{LKM}}=\mu_{1, \mathrm{EDM}}$. For continuous breakthrough curves, the first term on the right hand size of Eqs. (23) and (24) are zero. The effect of longitudinal diffusion is not significant with respect to retention time or first moment.

\section{Second central moment:}

The second central moment (variance) of the elution profile provides information about the rate of mass transfer process in the column. The second central moments for a rectangular profile is given as $[5,6,23]$ :

$$
\mu_{2, \mathrm{GRM}}^{\prime}=\frac{t_{\mathrm{inj}}^{2}}{12}+\frac{2 L D_{L}}{u^{3}}\left(1+a^{*} F\right)^{2}+\frac{2 L a^{*} F}{u}\left(\frac{R_{p} a^{*}}{3 k_{\mathrm{ext}}}+\frac{R_{p}^{2} a^{*}}{15 D_{\mathrm{eff}}}\right) .
$$

Eq. (25) reduces to the second central moment of LKM by using

$$
a^{*}=a, \quad\left(\frac{R_{p} a^{*}}{3 k_{\mathrm{ext}}}+\frac{R_{p}^{2} a^{*}}{15 D_{\mathrm{eff}}}\right)=\frac{1-\epsilon}{k_{\mathrm{LKM}}} .
$$

Here, $k_{\text {LKM }}$ is the mass transfer coefficient in the LKM model. Using the above relation in Eq. (25), we obtain

$$
\mu_{2, \mathrm{LKM}}^{\prime}=\frac{t_{\mathrm{inj}}^{2}}{12}+\frac{2 L D_{L}}{u^{3}}(1+a F)^{2}+\frac{2 L a F(1-\epsilon)}{u k_{\mathrm{LKM}}} .
$$

For $k_{\text {LKM }} \rightarrow \infty$, Eq. (27) reduces to the second central moments of EDM. For continuous breakthrough curves, the first terms on the right hand side of Eqs. (25) and (27) are zero. The first and second central moments can be used to analyze the flow rate dependence of Height Equivalent to Theoretical Plate (HETP) number. In order to use the moment expressions to derive the classical HETP-curve, let us consider for the sake of simplicity a Dirac injection, i.e. $t_{\text {inj }}=0$. Then, Eqs. (23), and (25) gives $[2,39]$

$$
\operatorname{HETP}_{\mathrm{GRM}}(u)=\frac{L \mu_{2}^{\prime}}{\mu_{1}^{2}}=\frac{2 D_{L}}{u}+\frac{2 F a^{* 2}}{\left(1+a^{*} F\right)^{2}}\left[\frac{R_{p}}{3 k_{\text {ext }}}+\frac{R_{p}^{2}}{15 D_{\text {eff }}}\right] u .
$$

In the above equation, the first term captures the band broadening by dispersion, the second term describes the contribution of external mass transfer, and the last term provides the contribution of diffusive migration of sample molecules inside the stationary phase. The first term is frequently splitted using the following simplifying expression [18]

$$
D_{L}=\gamma_{1} 2 R_{p} u+\gamma_{2} D_{\mathrm{mol}}
$$


Here, $D_{\text {mol }}$ is the molecular diffusivity of the solute in the solvent, while $\gamma_{1}$ and $\gamma_{2}$ represent weight factors for the relative impact of the particle size/flow rate dependent and the molecular diffusion based contributions to band broadening. Thus, Eq. (28) can be rewritten as

$$
\operatorname{HETP}_{\mathrm{GRM}}(u)=4 \gamma_{1} R_{p}+\frac{2 \gamma_{2} D_{\mathrm{mol}}}{u}+C u=A+\frac{B}{u}+C u,
$$

where

$$
A=4 \gamma_{1} R_{p}, \quad B=2 \gamma_{2} D_{\mathrm{mol}}, \quad C=\frac{2 F a^{* 2}}{\left(1+a^{*} F\right)^{2}}\left[\frac{R_{p}}{3 k_{\mathrm{ext}}}+\frac{R_{p}^{2}}{15 D_{\mathrm{eff}}}\right] .
$$

Eqs. (30) and (31) are the classical van Deemter equation (see [39]), valid only in this form for the general rate model using the simplifying Dirichlet $\mathrm{BC}$ and Dirac pulse injection. It should be mentioned here, that the $\mathrm{BC}$ related effect is often ignored in using and interpreting HETP-curves for comparing different chromatographic systems. For example in the case of Danckwerts BC, the $A$ - and $B$-terms in Eq. (31) change to [25]

$$
A=4 \gamma_{1} R_{p}\left[1+\frac{D_{L}}{L u}\left(e^{-\frac{L u}{D_{L}}}-1\right)\right], \quad B=2 \gamma_{2} D_{\mathrm{mol}}\left[1+\frac{D_{L}}{L u}\left(e^{-\frac{L u}{D_{L}}}-1\right)\right] .
$$

It can be easily observed that for a sufficiently small value of $D_{L}$, Eq. (32) reduces back to Eq. (31).

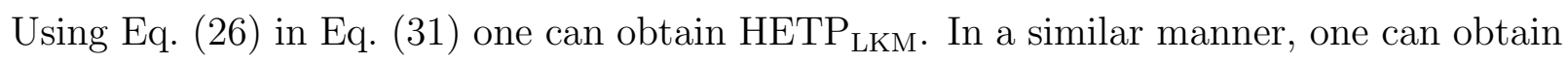
$\operatorname{HETP}_{\text {EDM }}$ by just letting $k_{\text {LKM }} \rightarrow \infty$ (i.e. $\mathrm{C}=0$ ).

\section{Third central moment:}

A peak shape is characterized by the third and fourth central moments. The analysis of peak shapes helps not only to gain qualitative and quantitative data, it explain the operation of the chromatographic system. Moreover, it could be helpful in investigating the mathematical parameters and mass-transfer characteristic of the chromatographic system. The peak skewness and kurtosis can be calculated from them which will be discussed below. The third central moment quantifies asymmetry of the elution profiles. It is given as 
$[5,6,23]:$

$$
\begin{aligned}
\mu_{3, \mathrm{GRM}}^{\prime}= & \frac{12 L D_{L}^{2}}{u^{5}}\left(1+a^{*} F\right)^{3}+\frac{12 L D_{L}\left(1+a^{*} F\right) F}{u^{3}}\left(\frac{R_{p} a^{* 2}}{3 k_{\mathrm{ext}}}+\frac{R_{p}^{2} a^{* 2}}{15 D_{\mathrm{eff}}}\right) \\
& +\frac{2 L a^{*} F}{u}\left(\frac{2 R_{p}^{4} a^{* 2}}{105 D_{\mathrm{eff}}^{2}}+\frac{2 R_{p}^{3} a^{* 2}}{15 k_{\mathrm{ext}} D_{\mathrm{eff}}}+\frac{R_{p}^{2} a^{* 2}}{3 k_{\mathrm{ext}}^{2}}\right) .
\end{aligned}
$$

Eq. (33) reduces to the third central moments of LKM by using Eq. (26) and

$$
\left(\frac{2 R_{p}^{4} a^{* 2}}{105 D_{\text {eff }}^{2}}+\frac{2 R_{p}^{3} a^{* 2}}{15 k_{\text {ext }} D_{\text {eff }}}+\frac{R_{p}^{2} a^{* 2}}{3 k_{\text {ext }}^{2}}\right)=\frac{3(1-\epsilon)^{2}}{k_{\text {LKM }}^{2}} .
$$

Using Eqs. (26) and (34) in Eq. (33), we obtain

$$
\mu_{3, \mathrm{LKM}}^{\prime}=\frac{12 L D_{L}^{2}}{u^{5}}(1+a F)^{3}+\frac{12 L D_{L}(1+a F) F(1-\epsilon)}{u^{3} k_{\mathrm{LKM}}}+\frac{6 L a F(1-\epsilon)^{2}}{u k_{\mathrm{LKM}}^{2}} .
$$

Note that $\mu_{3}^{\prime}$ is the same for rectangular and continuous breakthrough curves. For $k_{L K M} \rightarrow$ $\infty$, we obtain the third central moment of EDM.

The second and third central moments can be used to calculate the skewness that measures the degree of asymmetry of elution profiles. It can be evaluated as

$$
\delta_{\text {skew }}=\frac{\mu_{3}^{\prime}}{\left(\mu_{2}^{\prime}\right)^{3 / 2}} .
$$

This relation is useful to quantify deviations from Gaussian peak shapes.

As Eq. (36) shows, chromatographic peaks corresponding to GRM have some degree of asymmetry. Thus, their front and rare parts have not the same shape.

\section{Fourth central moment:}

Finally, the fourth central moment for rectangular concentration pulses of finite width is 
given as:

$$
\begin{aligned}
\mu_{4, \mathrm{GRM}}^{\prime}= & \frac{t_{\mathrm{inj}}^{4}}{80}+\frac{t_{\mathrm{inj}}^{2}}{u^{3}}\left[L D_{L}\left(1+a^{*} F\right)^{2}+L a^{*} F u^{2}\left(\frac{R_{p} a^{*}}{3 k_{\mathrm{ext}}}+\frac{R_{p}^{2} a^{*}}{15 D_{\mathrm{eff}}}\right)\right] \\
& +\frac{12 L^{2} D_{L}^{2}}{u^{6}}\left(1+a^{*} F\right)^{4}\left[\left(1+10 \frac{D_{L}}{L u}\right)\right] \\
& +\frac{24 L D_{L}^{2}\left(1+a^{*} F\right)^{2} a^{*} F}{u^{5}}\left(\frac{R_{p} a^{*}}{3 k_{\mathrm{ext}}}+\frac{R_{p}^{2} a^{*}}{15 D_{\mathrm{eff}}}\right)\left[6+\frac{L u}{D_{L}}\right] \\
& +\frac{4 L D_{L} a^{*} F}{u^{3}}\left(\frac{2 R_{p}^{4} a^{* 2}}{105 D_{\mathrm{eff}}^{2}}+\frac{2 R_{p}^{3} a^{* 2}}{15 k_{\mathrm{ext}} D_{\mathrm{eff}}}+\frac{R_{p}^{2} a^{* 2}}{3 k_{\mathrm{ext}}^{2}}\right)\left[4+a^{*} F\left(6+\frac{L u}{D_{L}}\right)\right] \\
& +\frac{8 L a^{*} F}{u}\left(\frac{1}{525} \frac{R_{p}^{6} a^{* 3}}{D_{\mathrm{eff}}^{3}}+\frac{3}{175} \frac{R_{p}^{5} a^{* 3}}{D_{\mathrm{eff}}^{2} k_{\mathrm{ext}}}+\frac{1}{15} \frac{R_{p}^{4} a^{* 3}}{D_{\mathrm{eff}} k_{\mathrm{ext}}^{2}}+\frac{1}{9} \frac{R_{p}^{3} a^{* 3}}{k_{\mathrm{ext}}^{3}}\right) \\
& -\frac{8 L a^{*} F}{175} \frac{F D_{L} a^{* 3} R_{p}^{4}}{u^{3} D_{\mathrm{eff}}^{2}}\left(1+\frac{L u}{2 D_{L}}\right) .
\end{aligned}
$$

Eq. (37) reduces to the fourth central moments of LKM by using Eqs. (26) and (34) along with

$$
\begin{aligned}
& \left(\frac{1}{525} \frac{R_{p}^{6} a^{* 3}}{D_{\mathrm{eff}}^{3}}+\frac{3}{175} \frac{R_{p}^{5} a^{* 3}}{D_{\mathrm{eff}}^{2} k_{\mathrm{ext}}}+\frac{1}{15} \frac{R_{p}^{4} a^{* 3}}{D_{\mathrm{eff}} k_{\mathrm{ext}}^{2}}+\frac{1}{9} \frac{R_{p}^{3} a^{* 3}}{k_{\mathrm{ext}}^{3}}\right) \\
& -\frac{1}{175} \frac{F D_{L} a^{* 3} R_{p}^{4}}{u^{2} D_{\mathrm{eff}}^{2}}\left(1+\frac{L u}{2 D_{L}}\right)=\frac{3(1-\epsilon)^{3}}{k_{\mathrm{LKM}}^{3}}
\end{aligned}
$$

Thus, we obtain

$$
\begin{aligned}
\mu_{4, \mathrm{LKM}}^{\prime}= & \frac{t_{\mathrm{inj}}^{4}}{80}+\frac{t_{\mathrm{inj}}^{2}}{u^{3}}\left[L D_{L}(1+a F)^{2}+\frac{L a F u^{2}(1-\epsilon)}{k_{\mathrm{LKM}}}\right]+\frac{12 L^{2} D_{L}^{2}}{u^{6}}(1+a F)^{4}\left[1+10 \frac{D_{L}}{L u}\right] \\
& +\frac{24 L D_{L}^{2}(1+a F)^{2} a F(1-\epsilon)}{k_{\mathrm{LKM}} u^{5}}\left[6+\frac{L u}{D_{L}}\right]+\frac{12 L D_{L} a F(1-\epsilon)^{2}}{k_{\mathrm{LKM}}^{2} u^{3}}\left[4+a F\left(6+\frac{L u}{D_{L}}\right)\right] \\
& +\frac{24 L a F(1-\epsilon)^{3}}{u k_{\mathrm{LKM}}^{3}}
\end{aligned}
$$

The fourth central moment of continuous breakthrough profiles can be obtained from Eq. (37) by putting all terms containing $t_{\text {inj }}$ equal to zero. For $k_{\mathrm{LKM}} \rightarrow \infty$, we obtain the fourth central moment of EDM.

The fourth central moment, i.e. kurtosis, measures the profiles peakedness or flatness relative to a normal distribution. In general, the kurtosis is a descriptor of the shape of a probability distribution. It is instructive to use an adjusted version of Pearson's kurtosis, 
the excess kurtosis [40]. The excess kurtosis compares the shape of a given distribution to that of the normal distribution. Distributions with negative or positive excess kurtosis are called platykurtic distributions or leptokurtic distributions, respectively. The following definition quantifies the excess kurtosis [40]

$$
\gamma_{\mathrm{kur}}=\frac{\mu_{4}^{\prime}}{\left(\mu_{2}^{\prime}\right)^{2}}-3
$$

A high kurtosis distribution has a sharper peak and a broader tails than the normal distribution, while a low kurtosis distribution has a more rounded peak and thinner tails. Distributions with zero excess kurtosis, as the normal distribution, are called mesokurtic. The fourth central moment associated with kurtosis is used to study the flatness of chromatogram elution.

\section{Moments of 1D GRM for core-shell particles}

The next part of this study is concerned with cored particles of arbitrary inert core radius $R_{\text {core }}$. For fully porous particles $r$ ranges from 0 to $R_{p}$, while for cored particles it ranges from $r=R_{\text {core }}$ to $R_{p}$ [25]. The first three moments are derived for GRM considering a column packed with core-shell particles [25]. The complete derivations of moments are presented below using the considered two types of boundary conditions.

\subsubsection{Type I: Dirichlet $B C$}

In this case, the moments up to third order are given below [25].

Zeroth moment: The zeroth moment for rectangular profiles is given as

$$
\mu_{0, \mathrm{GRM}}=c_{\mathrm{inj}} t_{\mathrm{inj}}
$$

The zeroth moment for continuous breakthrough curves is simply $\mu_{0, \mathrm{GRM}}=c_{\mathrm{inj}}$. Let us define

$$
\rho_{\text {core }}=R_{\text {core }} / R_{p}, \quad P e=\frac{L u}{D_{L}}, \quad B i=\frac{k_{\text {ext }} R_{p}}{D_{\text {eff }}}, \quad \eta=\frac{D_{\text {eff }} L}{R_{p}^{2} u}, \quad \xi=3 F \frac{k_{\text {ext }}}{R_{p}} \frac{L}{u} .
$$


Here, $R_{\text {core }}$ is the radius of the non-porous core and $R_{p}$ is the radius of the particle, Moreover, let us introduce

$$
\begin{aligned}
\tilde{a} & =\left(1-\rho_{\text {core }}^{3}\right) a^{*}, \quad \rho_{1}=\frac{\rho_{\text {core }}\left(1+3 \rho_{\text {core }}+3 \rho_{\text {core }}^{2}-\rho_{\text {core }}^{3}\right)}{\left(1+\rho_{\text {core }}+\rho_{\text {core }}^{2}\right)^{2}}, \\
\eta_{\text {mod }} & =\frac{\eta}{\rho_{\text {mod }}}, \quad \rho_{\text {mod }}=\left(1+\rho_{1}\right)\left(1-\rho_{\text {core }}\right)=\frac{1+2 \rho_{\text {core }}+3 \rho_{\text {core }}^{2}-\rho_{\text {core }}^{3}-5 \rho_{\text {core }}^{4}}{\left(1+\rho_{\text {core }}+\rho_{\text {core }}^{2}\right)^{2}} .
\end{aligned}
$$

Then, the remaining temporal moments are given below.

First moment: The first temporal moment for rectangular profiles is given as

$$
\mu_{1, \mathrm{GRM}}=\frac{t_{\mathrm{inj}}}{2}+\frac{L}{u}(1+\tilde{a} F) .
$$

Second central moment: The second central moment is given as

$$
\mu_{2, \mathrm{GRM}}^{\prime}=\frac{t_{\mathrm{inj}}^{2}}{12}+\frac{2 L D_{L}}{u^{3}}(1+\tilde{a} F)^{2}+\frac{2 L \tilde{a} F}{u}\left[\frac{R_{p} \tilde{a}}{3 k_{\mathrm{ext}}}+\frac{R_{p}^{2} \tilde{a}}{15 D_{\mathrm{eff}}} \rho_{\mathrm{mod}}\right] .
$$

Eqs. (45) and (46) for $\rho_{\text {core }}=0$ and $\rho_{\text {mod }}=1$ are identical to the moments of fully porous particles given by Eqs. (23) and (25).

To analyze breakthrough curves the first right hand side term in Eq. (46) needs to be set to zero.

In this case, the HETP can be obtained from Eq. (45) and (46) as given below:

$$
\operatorname{HETP}_{\text {GRM }}(u)=\frac{L \mu_{2}^{\prime}}{\mu_{1}^{2}}=4 \gamma_{1} R_{p}+\frac{2 \gamma_{2} D_{\text {mol }}}{u}+C_{\text {core }} u=A+\frac{B}{u}+C_{\text {core }} u,
$$

where

$$
A=4 \gamma_{1} R_{p}, \quad B=2 \gamma_{2} D_{\mathrm{mol}}, \quad C_{\text {core }}=\frac{2 F_{b} \tilde{a}^{2}}{\left(1+\tilde{a} F_{b}\right)^{2}}\left[\frac{R_{p}}{3 k_{\mathrm{ext}}}+\frac{R_{p}^{2}}{15 D_{\mathrm{eff}}} \rho_{\mathrm{mod}}\right] .
$$

For fully porous particles, i.e. $\rho_{\text {core }}=0, \tilde{a}=a^{*}$, and $\rho_{\text {mod }}=1$, the Eqs. (47) and (48) reduces to the classical van Deemter equation (see [39]) given by Eqs. (30) and (31), valid only in this form for the general rate model using the simplifying Dirichlet BC and Dirac pulse injection.

The minimum of HETP is found at the following optimal velocity:

$$
u_{\text {opt }}\left(\rho_{\text {core }}\right)=\sqrt{\frac{B}{C_{\text {core }}}} .
$$


For this velocity the following minimum of HETP results:

$$
\operatorname{HETP}_{\text {min }}\left(u_{\text {opt }}\left(\rho_{\text {core }}\right)\right)=A+2 \sqrt{B C_{\text {core }}} .
$$

Third central moment: The third central moment is calculated as

$$
\begin{aligned}
\mu_{3, \mathrm{GRM}}^{\prime}= & \frac{12 L D_{L}^{2}}{u^{5}}(1+\tilde{a} F)^{3}+\frac{6 L^{2} D_{L}(1+\tilde{a} F) F}{u^{4}}\left[\frac{2 F \tilde{a}^{2}}{\xi}+\frac{2 \tilde{a}^{2}}{15 \eta_{\text {mod }}}\right] \\
& +\frac{L^{3} F \tilde{a}^{3}}{u^{3}}\left[\frac{4}{105 \eta_{\text {mod }}^{2}} \frac{\left(1+\rho_{2}\right)}{\left(1+\rho_{1}\right)^{2}}+\frac{4 F}{5 \xi \eta_{\text {mod }}}+\frac{6 F^{2}}{\xi^{2}}\right]
\end{aligned}
$$

where

$$
\rho_{2}=\frac{\rho_{\text {core }}\left(2+9 \rho_{\text {core }}+\frac{35}{2} \rho_{\text {core }}^{2}+\frac{23}{2} \rho_{\text {core }}^{3}-3 \rho_{\text {core }}^{4}-\rho_{\text {core }}^{5}\right)}{\left(1+\rho_{\text {core }}+\rho_{\text {core }}^{2}\right)^{3}} .
$$

Moreover, $\mu_{3, \mathrm{GRM}}^{\prime}$ is the same for rectangular and continuous breakthrough curves.

\subsubsection{Type II: Danckwerts (or Robin) type BC}

In this case, the moments are given as follows.

Zeroth moment: The zeroth moment for rectangular profiles is again given as

$$
\mu_{0, \mathrm{GRM}}=c_{\mathrm{inj}} t_{\mathrm{inj}}
$$

For continuous breakthrough curves, holds $\mu_{0, \mathrm{GRM}}=c_{\mathrm{inj}}$.

First moment: The first temporal moment from the Laplace transform solution can be obtained as

$$
\mu_{1, \mathrm{GRM}}=\frac{t_{\mathrm{inj}}}{2}+\frac{L}{u}(1+\tilde{a} F)
$$

For continuous breakthrough curves, the first term on the right hand side of the above equation is zero.

Second central moment: The second central moment for a rectangular pulse injection is given as

$$
\mu_{2, \mathrm{GRM}}^{\prime}=\frac{t_{\mathrm{inj}}^{2}}{12}+\frac{2 L D_{L}(1+\tilde{a} F)^{2}}{u^{3}}\left[1+\frac{D_{L}}{L u}\left(e^{-\frac{L u}{D_{L}}}-1\right)\right]+\frac{2 L \tilde{a} F}{u}\left[\frac{R_{p} \tilde{a}}{3 k_{\mathrm{ext}}}+\frac{R_{p}^{2} \tilde{a}}{15 D_{\mathrm{eff}}} \rho_{\mathrm{mod}}\right] .
$$


For continuous breakthrough curves, the first term on the right hand side of Eq. (55) is zero. Eq. (46) results from Eq. (55) if there the term featuring the boundary conditions is set to 1 , which is acceptable only for large $D_{L}$, i.e. for larger flow rates.

The expressions for first and second central moments can be used to derive the HETP-curve for the Danckwerts BC, i.e.

$$
\operatorname{HETP}(u)=\frac{L\left(\mu_{2}^{\prime}\right)^{t^{2}}}{\left(\mu_{1}^{2}\right)^{t}}=\frac{2 D_{L}}{u}\left[1+\frac{D_{L}}{L u}\left(e^{-\frac{L u}{D_{L}}}-1\right)\right]+\frac{2 F \tilde{a}^{2}}{(1+\tilde{a} F)^{2}}\left[\frac{R_{p}}{3 k_{\mathrm{ext}}}+\frac{R_{p}^{2}}{15 D_{\mathrm{eff}}} \rho_{\mathrm{mod}}\right] u .
$$

Now inserting of expression in Eq. (29) provides a more complicated dependence of HETP on $u$ and $D_{L}$. In this case, the $A$ - and $B$-terms in Eq. (31) change to

$$
A=4 \gamma_{1} R_{p}\left[1+\frac{D_{L}}{L u}\left(e^{-\frac{L u}{D_{L}}}-1\right)\right], \quad B=2 \gamma_{2} D_{\mathrm{mol}}\left[1+\frac{D_{L}}{L u}\left(e^{-\frac{L u}{D_{L}}}-1\right)\right] .
$$

Thus, these terms are not this simple anymore. In contrast to the case of Diriclet BC, the determination of $\mathrm{HETP}_{\text {min }}$ and $u_{\mathrm{opt}}$ requires numerical methods. Hereby, HETP-values corresponding to Dankwerts BC will be always less than the HETP for Diriclet BC.

Third central moment: The third central moment for a rectangular pulse injection is expressed as

$$
\begin{aligned}
\mu_{3, \mathrm{GRM}}^{\prime}= & \frac{12 L D_{L}^{2}(1+\tilde{a} F)^{3}}{u^{5}}\left[\left(1+\frac{2 D_{L}}{L u}\right) e^{-\frac{L u}{D_{L}}}+\left(1-\frac{2 D_{L}}{L u}\right)\right]+\frac{\tilde{a}^{3} F L^{3}}{u^{3}}\left(\frac{4}{105 \eta_{\mathrm{mod}}^{2}} \frac{\left(1+\rho_{2}\right)}{\left(1+\rho_{1}\right)^{2}}\right. \\
& \left.+\frac{4 F}{5 \xi \eta_{\mathrm{mod}}}+\frac{6 F^{2}}{\xi^{2}}\right)+\frac{6 L^{2} D_{L} F(1+\tilde{a} F)}{u^{4}}\left(\frac{2 F \tilde{a}^{2}}{\xi}+\frac{2 \tilde{a}^{2}}{15 \eta_{\mathrm{mod}}}\right)\left[\frac{D_{L}}{L u}\left(e^{-\frac{L u}{D_{L}}}-1\right)+1\right] .
\end{aligned}
$$

The third central moment is same for continuous breakthrough curve. Note that, Eq. (51) is a special case of Eq. (58) valid for large $D_{L}$ numbers for which the Danckwerts BC reduces into the Dirichlet $\mathrm{BC}$.

\section{Moments of 2D GRM for fully porous particles}

In the more difficult 2D case, the analytical temporal moments are obtained in the Hankel domain as functions of the dimensionless radial coordinate $\psi=\rho / R_{\text {core }}$ [25]. The true 
moments $\mu_{i}(\psi)$ are generated by using the following expression [24]

$$
\mu_{i}(\psi)=2 \mu_{i, H}\left(\lambda_{n}=0\right)+2 \sum_{n=1}^{\infty} \mu_{i, H}\left(\lambda_{n}\right) \frac{J_{0}\left(\lambda_{n} \psi\right)}{\left|J_{0}\left(\lambda_{n}\right)\right|^{2}},
$$

where $\lambda_{n}$ is the finite Hankel transform parameter as determined by the transcendental equation $\frac{d J_{0}\left(\lambda_{n}\right)}{d \psi}=-J_{1}\left(\lambda_{n}\right)=0$. Here, $J_{0}($.$) and J_{1}($.$) are the zeroth and first order Bessel$ functions of the first kind. The averaged non-normalized temporal moments $M_{i, \text { av }}$ can be calculated as

$$
M_{i, \mathrm{av}}=2 \int_{0}^{1} \mu_{i}(\psi) \psi d \psi, \quad i=0,1,2, \cdots .
$$

The normalized averaged temporal moments used in chemical engineering are given as [2]:

$$
\mu_{i, \mathrm{av}}=\frac{M_{i, \mathrm{av}}}{\mu_{0, \mathrm{av}}}, \quad \mu_{0, \mathrm{av}}=M_{0, \mathrm{av}}, \quad i=1,2,3, \cdots .
$$

The above temporal moments $\mu_{i \text {,av }}$ up to the fourth order are obtained for interpretation of the solute transport behavior. These moments can be used to obtain the first four central moments defined in Eqs. (18)- (20).

In the following, the first four dimensionless Hankel domain moments are presented using Dirichlet BCs. Let us define

$$
P e_{\rho}=\frac{R^{2} u}{D_{\rho} L}, \quad \tau=\frac{u t}{L}, \quad \tilde{\psi}=\tilde{\rho} / R .
$$

Zeroth moment: It is given as

$$
\mu_{0, H}=\tau_{\text {inj }} F\left(\lambda_{n}\right) e^{-P e\left(\frac{w-1}{2}\right)},
$$

where

$$
w=\sqrt{1+\frac{4 \lambda_{n}^{2}}{P e P e_{\rho}}} .
$$

For injection at the inner cylindrical core, $F\left(\lambda_{n}\right)$ is given as

$$
F\left(\lambda_{n}\right)=\left\{\begin{array}{lr}
\frac{\tilde{\psi}^{2}}{2}, & \text { if } \lambda_{n}=0 \\
\frac{\tilde{\psi}}{\lambda_{n}} J_{1}\left(\lambda_{n} \tilde{\psi}\right), & \text { if } \lambda_{n} \neq 0
\end{array}\right.
$$


and for injection at the outer annular ring, we have it as:

$$
F\left(\lambda_{n}\right)= \begin{cases}\left(\frac{1}{2}-\frac{\tilde{\psi}^{2}}{2}\right), & \text { if } \lambda_{n}=0 \\ -\frac{\tilde{\psi}}{\lambda_{n}} J_{1}\left(\lambda_{n} \tilde{\psi}\right), & \text { if } \lambda_{n} \neq 0\end{cases}
$$

First moment: The First temporal moment is calculated as:

$$
\mu_{1, H}=\left[\frac{\tau_{\text {inj }}}{2}+\frac{1+a^{*} F}{w}\right] \mu_{0, H}
$$

Second moment: The second temporal moment is given as:

$$
\mu_{2, H}=\left[\frac{\tau_{i n j}^{2}}{3}+\frac{\left(1+a^{*} F\right)}{w} \tau_{i n j}+\frac{\left(1+a^{*} F\right)^{2}}{w^{2}}+\frac{2\left(1+a^{*} F\right)^{2}}{P e w^{3}}+\frac{2 a^{*^{2}} F\left(B_{i}+5\right)}{15 w \eta B_{i}}\right] \mu_{0, H} .
$$

Third moment: The third temporal moment is expressed as:

$$
\begin{aligned}
\mu_{3, H}= & {\left[\frac{\tau_{\text {inj }}^{3}}{4}+\frac{\left(1+a^{*} F\right)}{w} \tau_{\text {inj }}^{2}+\left(\frac{3\left(1+a^{*} F\right)^{2}}{2 w^{2}}+\frac{a^{* 2} F\left(B_{i}+5\right)}{5 w B_{i} \eta}\right.\right.} \\
& \left.+\frac{3\left(1+a^{*} F\right)^{2}}{P e w^{3}}\right) \tau_{i n j}+\frac{4 a^{*} F\left(\frac{35}{2}+7 B_{i}+B_{i}^{2}\right)}{105 w B_{i}^{2} \eta^{2}} \\
& +\frac{12\left(1+a^{*} F\right)^{3}}{P e^{2} w^{5}}+\frac{4 a^{* 2} F\left(1+a^{*} F\right)\left(B_{i}+5\right)}{5 P e w^{3} B_{i} \eta} \\
& \left.+\frac{6\left(1+a^{*} F\right)^{3}}{P e w^{4}}+\frac{2 a^{* 2} F\left(1+a^{*} F\right)\left(B_{i}+5\right)}{5 w^{2} B_{i} \eta}+\frac{\left(1+a^{*} F\right)^{3}}{w^{3}}\right] \mu_{0, H} .
\end{aligned}
$$


Fourth moment: The fourth temporal moment is given as:

$$
\begin{aligned}
\mu_{4, H}= & {\left[\frac{\tau_{\text {inj }}^{4}}{5}+\frac{\left(1+a^{*} F\right)}{w} \tau_{\text {inj }}^{3}+\left(\frac{4\left(1+a^{*} F\right)^{2}}{P e w^{3}}+\frac{4 a^{* 2} F\left(B_{i}+5\right)}{15 w B_{i} \eta}+\frac{2\left(1+a^{*} F\right)^{2}}{w^{2}}\right) \tau_{\text {inj }}^{2}\right.} \\
& +\left(\frac{2\left(1+a^{*} F\right)^{3}}{w^{3}}+\frac{8 a^{* 2} F\left(1+a^{*} F\right)\left(B_{i}+5\right)}{5 P e w^{3} B_{i} \eta}+\frac{8 a^{* 3} F\left(\frac{35}{2}+7 B_{i}+B_{i}^{2}\right)}{105 w B_{i}^{2} \eta^{2}}\right. \\
& \left.+\frac{24\left(1+a^{*} F\right)^{3}}{P e^{2} w^{5}}+\frac{12\left(1+a^{*} F\right)^{3}}{P e w^{4}}+\frac{4 a^{* 2} F\left(1+a^{*} F\right)\left(B_{i}+5\right)}{5 w^{2} B_{i} \eta}\right) \tau_{\text {inj }} \\
& +\frac{32 a^{* 3} F\left(1+a^{*} F\right)\left(\frac{35}{2}+7 B_{i}+B_{i}^{2}\right)}{105 P e w^{3} B_{i}^{2} \eta^{2}}+\frac{48 a^{* 2} F\left(1+a^{*} F\right)^{2}\left(B_{i}+5\right)}{5 P e^{2} w^{5} B_{i} \eta}+\frac{\left(1+a^{*} F\right)^{4}}{w^{4}} \\
& +\frac{8 a^{* 4} F^{2}\left(B_{i}+5\right)^{2}}{75 P e w^{3} B_{i}^{2} \eta^{2}}+\frac{24 a^{* 2} F\left(1+a^{*} F\right)^{2}\left(B_{i}+5\right)}{5 P e w^{4} B_{i} \eta}+\frac{4 a^{* 4} F^{2}\left(B_{i}+5\right)^{2}}{75 w^{2} B_{i}^{2} \eta^{2}} \\
& +\frac{120\left(1+a^{*} F\right)^{4}}{P e w^{7}}+\frac{8 a^{* 3} F\left(1+a^{*} F\right)\left(\frac{35}{2}+7 B_{i}+B_{i}^{2}\right)}{105 w^{2} B_{i}^{2} \eta^{2}}+\frac{60\left(1+a^{*} F\right)^{4}}{P e^{2} w^{6}} \\
& \left.+\frac{12\left(1+a^{*} F\right)^{4}}{P e w^{5}}+\frac{4 a^{* 2} F\left(1+a^{*} F\right)^{2}\left(B_{i}+5\right)}{5 w^{3} B_{i} \eta}+\frac{8 a^{* 4} F\left(175+105 B_{i}+27 B_{i}^{2}\right)}{1575 w B_{i}^{3} \eta^{3}}\right] \mu_{0, H} .
\end{aligned}
$$

\section{Numerical Test Problems}

In this section, selected analytical moments are analyzed corresponding to the more general GRM by considering several test problems. For comparison, a second-order accurate finite volume scheme (FVS) of Koren was chosen to solve model equations of GRM numerically and to calculate the moments by integrating the obtained concentration profiles [26]. The normalized $n$-th temporal moment of the band profile at the outlet of a column of length $L$ is given as

$$
\mu_{n}=\frac{\int_{0}^{\infty} c(t, L) t^{n} d t}{\int_{0}^{\infty} c(t, L) d t} .
$$

While, the corresponding $n$-th central moment is expressed as

$$
\mu_{n}^{\prime}=\frac{\int_{0}^{\infty} c(t, L)\left(t-\mu_{1}\right)^{n} d t}{\int_{0}^{\infty} c(t, L) d t}
$$

The trapezoidal rule was applied to approximate the integrals in Eqs. (71) and (72). Eqs. (71) and (72) were also used to evaluate moments of the solutions obtained by taking numerical inversion of the analytical Laplace domain solutions. 


\subsection{Test cases for fully porous particles}

Figure 2 shows a comparison of LKM moments for two different values of $k_{\text {LKM }}$ with those of GRM. It can be seen that for small values of $k_{\mathrm{LKM}}$, the second, third and third central moments of LKM have large values as compared to GRM. On the other hand, for large values of $k_{\mathrm{LKM}}$ (i.e. for EDM), the central moments of GRM have large values. This means that for small values of $k_{\mathrm{LKM}}$, elution profiles of LKM are broader. The standard parameters used in the test problems are given in Table 1.

A quantitative comparison of skewness and kurtosis of GRM and LKM is given in Figure 3 over $u$ using Danckwerts BC. The skewness for a normal distribution is zero, and any symmetric data should have a skewness near zero. Negative values of the skewness indicate that data are left skewed and positive values indicate the right skewed data. It can be observed that the Kurtosis of LKM approaches to that of GRM when $k_{\mathrm{LKM}}$ is increased. Figure 4 shows the comparison of moments obtained from GRM for the considered two sets of boundary conditions using two different values of $D_{L}$. It can be observed that moments of both boundary conditions agree well for small value of axial dispersion, $D_{L}=$ $0.002 \mathrm{~cm}^{2} / \mathrm{min}$, but are significantly different for large values of axial dispersion, $D_{L}=$ $1.0 \mathrm{~cm}^{2} / \mathrm{min}$. Thus, for large axial dispersion, Danckwerts $\mathrm{Bc}$ is more accurate which accounts for back mixing in the vicinity of column inlet.

\subsection{Test cases for core-shell particles}

In this section, the effect of $\rho_{\text {core }}$, characterizing the extension of the inert core, on the the moments is analyzed. The same finite volume scheme is chosen to obtain numerical moments [26]. The standard parameters used in the test problems are given in Table 1. A quantitative comparison of analytical and numerical moments for different core radius fractions is presented in Figure 5 considering considering Danckwerts BCs. As $\rho_{\text {core }}$ increases from 0 (fully porous beads) to 0.8 (beads with a thin shell), the values of moments are decreasing. With an increase of $\rho_{\text {core }}$, the first moment $\mu_{1}$, describing the retention time of the elution profile, decreases. As $\rho_{\text {core }}$ increases, the profiles become sharper, giving a reduction in the second central moment $\mu_{2}^{\prime}$ which quantifies the variance of the concen- 
tration profile. Further, the third moment, which quantifies the asymmetry of the elution profiles, is also decreasing with increasing $\rho_{\text {core }}$. The good agreement between analytical and numerical results demonstrates both the correctness of analytical calculations and high precision of FVS.

Probably as the most interesting result for chromatography petitioners, in Figure 6 are plotted the plate heights HETP (c.f. Eq. (47)-(50)) and reduced plate heights $h$ as functions of the velocity $u$. The illustrating calculations were done for the Dirichlet BC and different fractions of the nonporous core assuming in all cases a column length of $L=20 \mathrm{~cm}$, a particle radius of $R_{p}=2 \times 10^{-4} \mathrm{~cm}, \gamma_{1}=0.5, \gamma_{2}=1, D_{\mathrm{mol}}=6 \times 10^{-4} \mathrm{~cm}^{2} / \mathrm{min}$, $k_{\text {ext }}=5 \mathrm{~cm} / \mathrm{min}, D_{L}=0.1 \mathrm{~cm}^{2} / \min$ and $D_{\text {eff }}=2 \times 10^{-5} \mathrm{~cm}^{2} / \mathrm{min}$. It can be seen that an increase in $\rho_{\text {core }}$, i.e. a decrease of the thickness of the porous layer, causes smaller HETP-values. For the conditions chosen, i.e. for a rather efficient column, i.e. not severe back-mixing, there is no visible difference in using Diriclet (Eqs. (47) and (48)) or the more realistic Danckwerts boundary conditions (Eqs. (56) and (57)). The optimum velocity $u_{\text {opt }}$ and minima of HETP (c.f. Eqs. (49) and (50)) are also plotted over different core radius fractions $\rho_{\text {core }}$. For larger $\rho_{\text {core }}$ the minima of HETP decrease and the corresponding flowrates increase. Thus, comparing the conditions offering the highest efficiencies, it can be concluded that core-shell particles can be operated at larger flow rates. This offers the attractive option for reducing the analysis times.

In order to quantify the effect of the type of $\mathrm{BC}$ on the HETP-values, the following ratio is introduced:

$$
\mathrm{BC}_{\text {HETP }}=\frac{\text { HETP for Dirichlet BC }}{\text { HETP for Danckwerts BC }} \text {. }
$$

To illustrate differences due to the application of the two types of boundary conditions, HETP and the ratio $\mathrm{BC}_{\text {HETP }}$ are plotted in Figure 7 as a functions of the velocity $u$ in a reduced velocity range before the minimum occurs. In contrast to the calculations presented in Figure 6 larger $\gamma_{2}$ (or $B$-term) and thus larger $D_{L}$ are used describing the performance of a less efficient column. For low efficient columns, i.e. small Pe numbers (low flowrates, short columns, large back-mixing effects), the selection of boundary conditions influences 
the results and the more realistic Danckwerts BC and the corresponding solutions should be applied. If $D_{L}$ is very small Eq. (56) converges into Eq. (28) and $\mathrm{BC}_{\mathrm{HETP}}$ becomes unity. HETP is smaller for the Dankweets BC due to the joint effects of the different inlet and out boundary conditions. For these rate parameters the core radius fraction $\rho_{\text {core }}$ was found to have no influence on HETP due to the dominance of the extraparticle back-mixing effect. This situation can change for other relative contributions of the mechanisms accounted for in the general rate model.

\subsection{Test problems for $2 D$ GRM}

Figure 8 displays the 2D local moments plotted along the radial coordinate of the column. The effect of radial dispersion coefficient on the first second, third and fourth moments can be clearly seen. Here, $P e=60, B_{i}=50$ and $\eta=2$ were kept fixed and varied was the ratio

$P e_{\text {ratio }}=\frac{P e_{\rho}}{P e}$ which corresponds. The plots of this figure show that moments approach to constant values along the radial coordinate for smallest value of $P e_{\text {ratio }}$ or largest $D_{r}$. For the smallest value of $P e_{\text {ratio }}=0.025$, the results correspond to the $1 \mathrm{D}$ results presented above. Since the concentration is injected via the inner cylindrical core, all moments do not change close to the column center. The changes clearly occur in the outer section. Although the trends look similar, on inspecting closer the $y$-axis, the magnitudes reveal that higher moment change more significantly with changing the $P e_{\text {ratio. Similar trends }}$ were also observed in the case of injection through outer zone.

\section{Conclusion}

Accurate and quantitative information about the dynamics in a chromatographic columns could be helpful to design appropriate separation systems and packing materials. In this review, the moment analysis (MA) method was introduced as an attractive technique for quantitatively analyzing the chromatographic process. This well-established method condenses the information provided by a chromatogram into a relative small number of temporal moments. MA could be an effective techniques to estimate free thermodynamic 
and kinetic model parameters from measured chromatograms. Due to its moment generating property, the Laplace domain solution can be used as basic tool to derive analytical expressions of temporal moments. A summary of temporal moments derived just recently for two standard liquid chromatographic models has been provided. We mainly focused on the effects of different boundary conditions, on the derivation of moment expressions up to fourth order for fully porous particles, on the derivation of the first three moments for core-shell particles, and on the derivation of the first four temporal moments of the 2D GRM. The derived moments could be a useful tool for understanding the chromatographic process and to analyze of the retention times and peak shapes. These theoretical moments could also be very helpful a) to describe in a simpler manner essential feature of the chromatograms, b) to estimate efficiently free model parameters by matching measured and predicted moments and c) to predict performance parameters of the separations and, thus, d) to optimize more easily the process.

\section{References}

[1] G. Guiochon, B. Lin, Modelling for preparative chromatography, Academic Press, Amsterdam, 2003.

[2] G. Guiochon, A. Felinger, D.G. Shirazi, A.M. Katti, Fundamentals of preparative and nonlinear chromatography, 2nd ed, Elsevier Academic press, New York, 2006.

[3] G. Guiochon, Preparative liquid chromatography. J. Chromatogr. A 965 (2002) 129-161.

[4] F. Gritti, G. Guiochon, Mass transfer kinetics, band broadening and column efficiency, J. Chromatogr. A 1221 (2012) 2-40.

[5] H. Gao, P.G. Stevenson, F. Gritti, G. Guiochon, Investigations on the calculation of the third moments of elution peaks. I: Composite signals generated by adding up a mathematical function and experimental noise, J. Chromatogr. A, 1222 (2012) 81-89. 
[6] H. Gao, F. Gritti, G. Guiochon, Investigations on the calculation of the third moments of elution peaks. II-Linear flow speed dependence of external mass transfer coefficient, J. Chromatogr. A, 1294 (2013) 41-49.

[7] K. Miyabe, G. Guiochon, Influence of the modification conditions of alkyl bonded ligands on the characteristics of reversed-phase liquid chromatography, J. Chromatogr. A 903 (2000) 1-12.

[8] K. Miyabe, G. Guiochon, Measurement of the parameters of the mass transfer kinetics in high performance liquid chromatography, J. Sep. Sci. 26 (2003) 155-173.

[9] K. Miyabe, Y. Matsumoto, G. Guiochon, Peak parking-moment analysis. A strategy for the study of the mass-transfer kinetics in the stationary phase, Anal. Chem. 79 (2007) 1970-82.

[10] K. Miyabe, Moment analysis of chromatographic behavior in reversed-phase liquid chromatography, J. Sep. Sci. 32 (2009) 757-770.

[11] M. Kubin, Beitrag zur Theorie der Chromatographie, Sorbens-Korns, Collect. Czech. Chem. C. 30 (1965) 1104-1118.

[12] M. Kubin, Beitrag zur Theorie der Chromatographie. 11. Einfluss der Diffusion Ausserhalb und der Adsorption Innerhalb des Sorbens- Korns, Collect. Czech. Chem. C. 30 (1965) 2900-2907.

[13] E. Kucera, Contribution to the theory of chromatography: Linear non-equilibrium elution chromatography, J. of Chromatogr. A 19 (1965) 237-248.

[14] P. Schneider, J.M. Smith, Adsorption rate constants from chromatography, AIChE. J, 14 (1968) 762-771.

[15] M. Suzuki, Notes on Determining the Moments of the Impulse Response of the Basic Transformed Equations, J. Chem. Eng. Japan 6 (1973) 540-543. 
[16] H.-J. Wolff, K.-H. Radeke, D. Gelbin, Heat and mass transfer in packed beds-IV use of weighted moments to determine axial dispersion coefficient, Chem. Eng. Sci. 34 (1980) 101-107.

[17] H.-J. Wolff, K.-H. Radeke, D. Gelbin, Weighted moments and the pore-diffusion model, Chem. Eng. Sci. 35 (1980) 1481-1485.

[18] D.M. Ruthven, Principles of adsorption and adsorption processes, Wiley-Interscience, New York, 1984.

[19] A.M. Lenhoff, Significance and estimation of chromatographic parameters, J. of Chromatogr. A 384 (1987) 285-299.

[20] G.M. Zhong, F. Meunier, G. Guiochon, Interference theory: Moment solution for two component adsorption chromatography, Chromatographia 36 (1993) 289-292.

[21] S. Javeed, S. Qamar, W. Ashraf, A. Seidel-Morgenstern, G. Warnecke, Analysis and numerical investigation of two dynamic models for liquid chromatography, Chem. Eng. Sci. 90 (2013) 17-31.

[22] S. Qamar, J.N. Abbasi, S. Javeed, M. Shah, F.U. Khan, A. Seidel-Morgenstern, Analytical solutions and moment analysis of chromatographic models for rectangular pulse injections, J. of Chromatogr. A 1315 (2013) 92-106.

[23] S. Qamar, J.N. Abbasi, S. Javeed, A. Seidel-Morgenstern, Analytical solutions and moment analysis of general rate model for linear liquid chromatography, Chem. Eng. Sci. 107 (2014) 192-205.

[24] S. Qamar, F.U. Khan, Y. Mehmood, A. Seidel-Morgenstern, Analytical solution of a two-dimensional model of liquid chromatography including moment analysis, Chem. Eng. Sci. 116 (2014) 576-589. 
[25] S. Qamar, J.N. Abbasi, A. Mehwish, A. Seidel-Morgenstern, Linear general rate model of chromatography for core-shell particles: Analytical solutions and moment analysis, Chem. Eng. Sci. 137 (2015) 352-363.

[26] S. Javeed, S. Qamar, A. Seidel-Morgenstern, G. Warnecke, Efficient and accurate numerical simulation of nonlinear chromatographic processes. Comput. Chem. Eng. 35 (2011) 2294-2305.

[27] C.G. Horvath, B.A. Preiss, S.R. Lipsky, Fast liquid chromatography: an investigation of operating parameters and the separation of nucleotides on pellicular ion exchangers, Anal. Chem. 39 (1967) 1422-1428.

[28] J.J. Kirkland, F.A. Truszkowski, C.H. Dilks, G.S. Engel, Superficially porous silica microspheres for fas thigh-performance liquid chromatography of macromolecules, J. Chromatogr. A 890 (2000) 3-13.

[29] J.J. Kirkland, T.J. Langlois, J.J. DeStefano, Fused core particles for HPLC columns, Am. Lab. 39 (2007) 18-21.

[30] F. Gritti, I. Leonardis, D. Shock, P. Stevenson, A. Shalliker, G. Guiochon, Performance of columns packed with the new shell particles, Kinetex-C18, J. Chromatogr. A 1217 (2010) 1589-603.

[31] F. Gritti, G. Guiochon, Comparison of heat friction effects in narrow-bore columns packed with core-shell and totally porous particles, Chem. Eng. Sci. 65 (2010) 6310-6319.

[32] F. Gritti, G. Guiochon, Facts and legends about columns packed with sub-3- $\mu m$ coreshell particles, LCGC North America 30 (2012) 586-595.

[33] F. Gritti, G. Guiochon, Effect of parallel segmented flow chromatography on the height equivalent to a theoretical plate II-Performances of $4.6 \mathrm{~mm} \times 30 \mathrm{~mm}$ columns packed with 2.6 $\mu m$ Accucore-C18 superficially porous particles, J. Chromatogr. A 1314 (2013) 44-53. 
[34] R. Hayes, A. Ahmed, T. Edge, H. Zhang, Coreshell particles: Preparation, fundamentals and applications in high performance liquid chromatography, J. Chromatogr. A 1357 (2004) 36-52.

[35] D.A. Spudeit, M.D. Dolzan, Z.S. Breitbach, W.E. Barber, G.A. Micke, D.W. Armstrong, Superficially porous particles vs. fully porous particles for bonded high performance liquid chromatographic chiral stationary phases: isopropyl cyclofructan 6 , J. Chromatogr. A 1363 (2104) 89-95.

[36] P.V. Danckwerts, Continuous flow systems, Chem. Eng. Sci. 2 (1953) 1-9.

[37] M. Suzuki, J.M. Smith, Kinetic studies by chromatography, Chem. Eng. Sci. 26 (1971) 221-235.

[38] Th. Van der Laan, Letter to the Editors on Notes on the diffusion type model for the longitudinal mixing in flow, Chem. Eng. Sci. 7 (1958) 187-191.

[39] J.J. Van Deemter, F.J. Zuiderweg, A. Klinkenberg, Longitudinal diffusion and resistance to mass transfer as causes of nonideality in chromatography, Chem. Eng. Sci. 5 (1956) 271-289.

[40] L.T. DeCarlo, On the meaning and use of kurtosis, Psychol. Methods 2 (1997) 292-307. 
Table 1: Parameters of the problems.

\begin{tabular}{|c|c|}
\hline Parameters & values \\
\hline Column length & $L=20 \mathrm{~cm}$ \\
External porosity & $\epsilon=0.4$ \\
Internal porosity & $\epsilon_{p}=0.333$ \\
Concentration at inlet & $c_{\text {inj }}=1.0 \mathrm{~g} / \mathrm{l}$ \\
Injection time & $t_{\text {inj }}=50 \mathrm{~min}$ \\
Adsorption equilibrium constant & $a=4.0$ \\
\hline
\end{tabular}




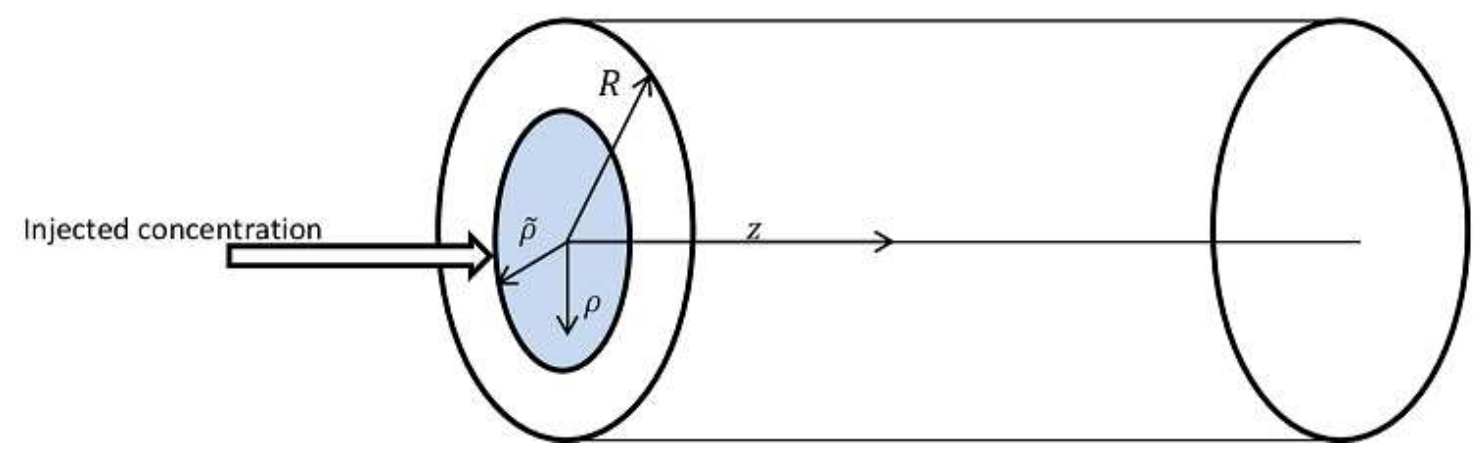

Figure 1: Schematic representation of a cylindrical column of cylindrical geometry [24]. 

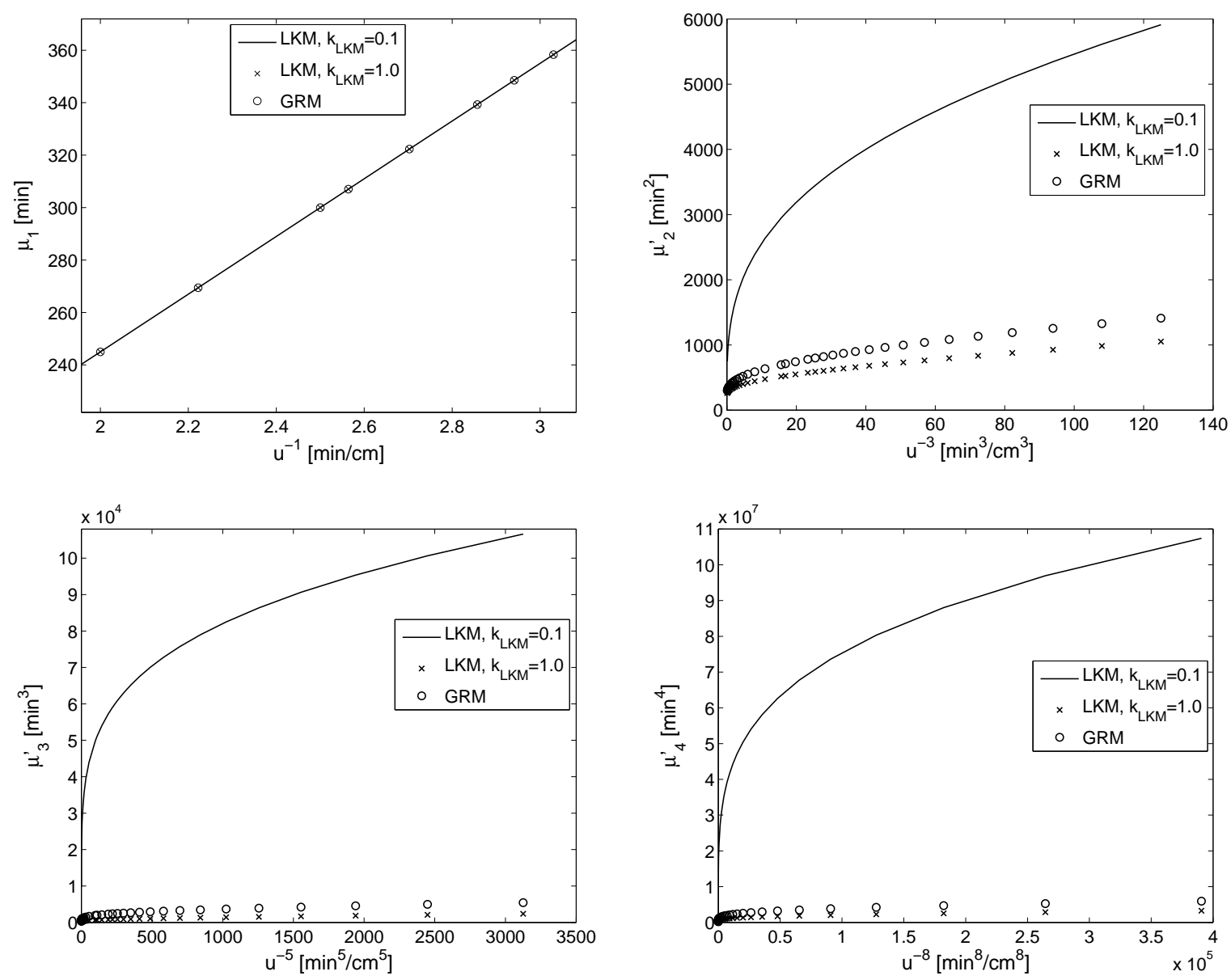

Figure 2: A comparison of LKM and GRM moments for $D_{L}=0.002 \mathrm{~cm}^{2} / \mathrm{min}, D_{\text {eff }}=10^{-6} \mathrm{~cm}^{2} / \mathrm{min}$, $R_{p}=0.002 \mathrm{~cm}, k_{\text {ext }}=0.01 \mathrm{~min}^{-1}$, and other parameters are given in Table 1 [23]. 

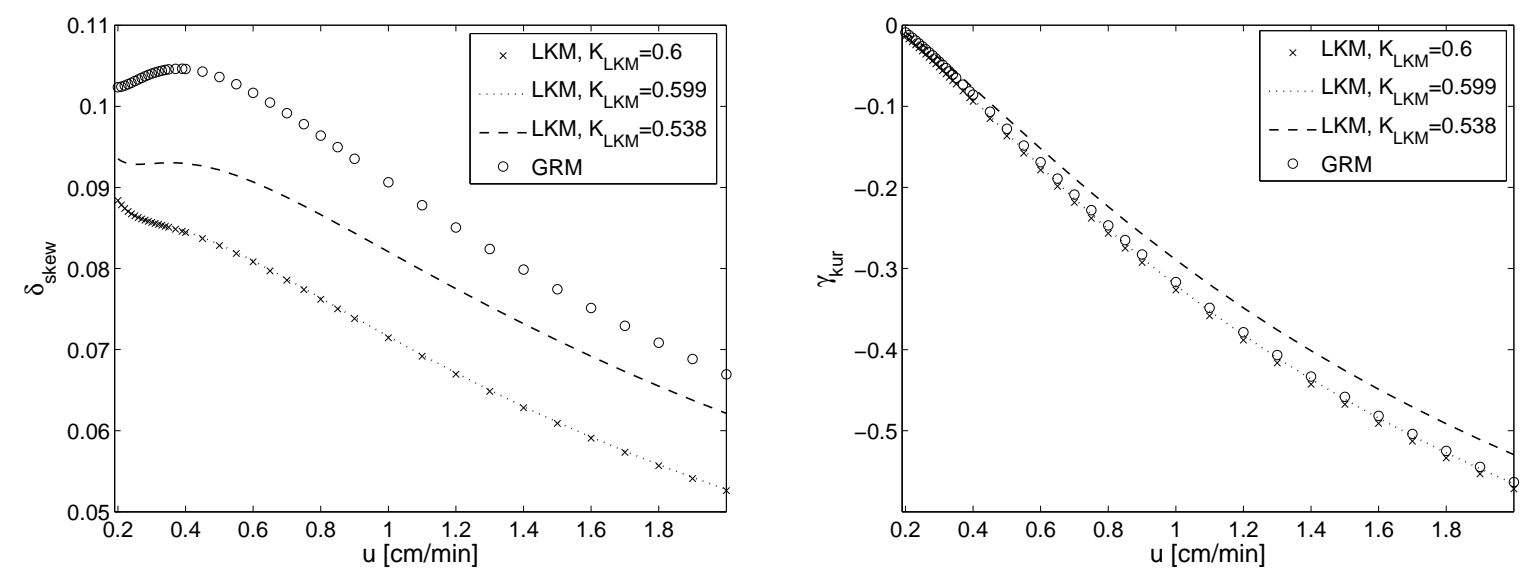

Figure 3: Skewness and kurtosis of GRM and LKM associated with third and fourth central moments considering different velocities $u$ [23]. Here, $D_{L}=0.002 \mathrm{~cm}^{2} / \min , D_{\text {eff }}=10^{-6} \mathrm{~cm}^{2} / \min , R_{p}=0.002 \mathrm{~cm}$ and $k_{\text {ext }}=0.01 \mathrm{~min}^{-1}$. All other parameters are given in Table 1 . 

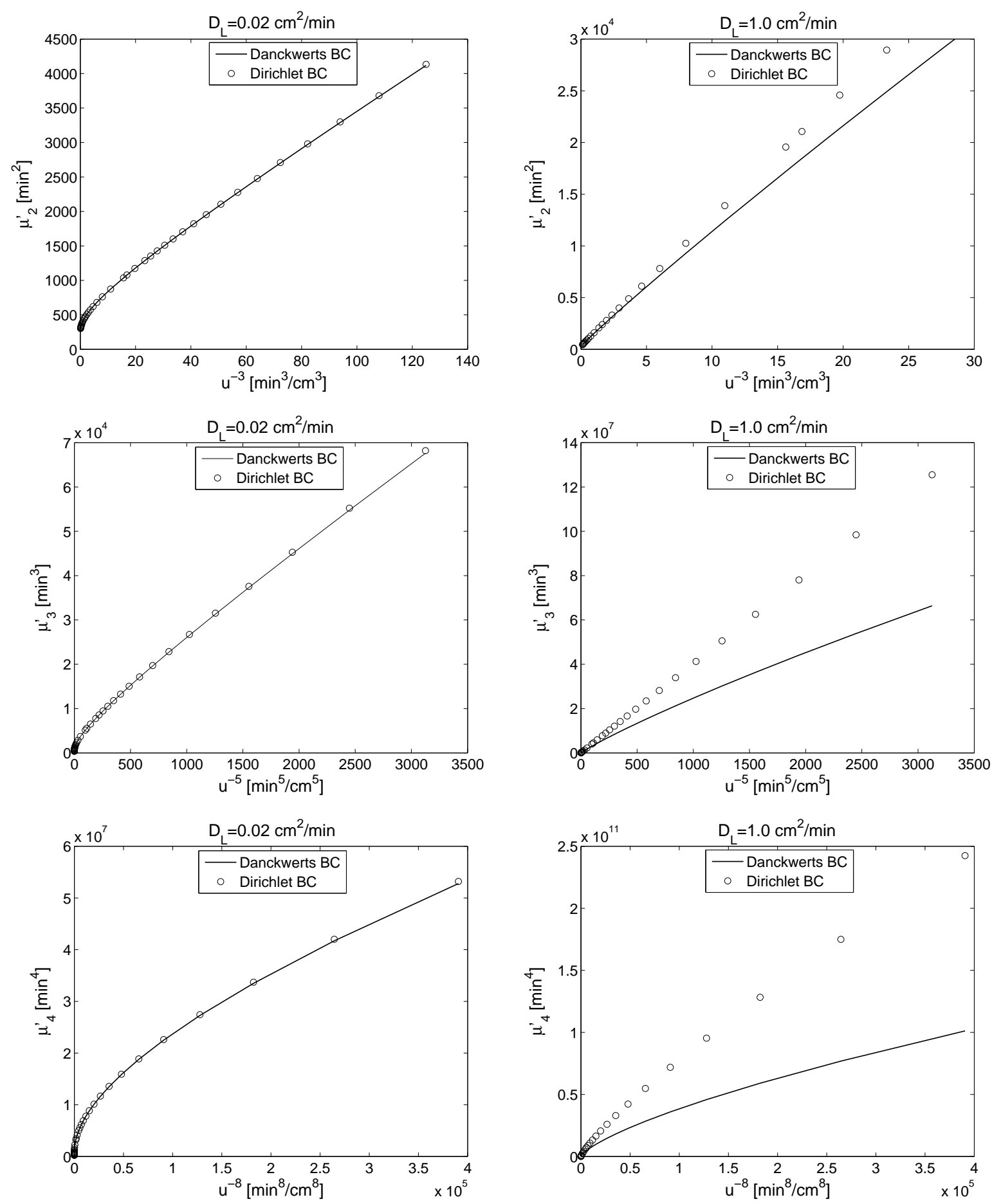

Figure 4: Moments of GRM for Danckwerts and Dirichlet BCs using two different values of $D_{L}$ [23]. Here, $D_{\text {eff }}=10^{-6} \mathrm{~cm}^{2} / \mathrm{min}, R_{p}=0.002 \mathrm{~cm}, k_{\text {ext }}=0.01 \mathrm{~min}^{-1}$ and other parameters are given in Table 1. 

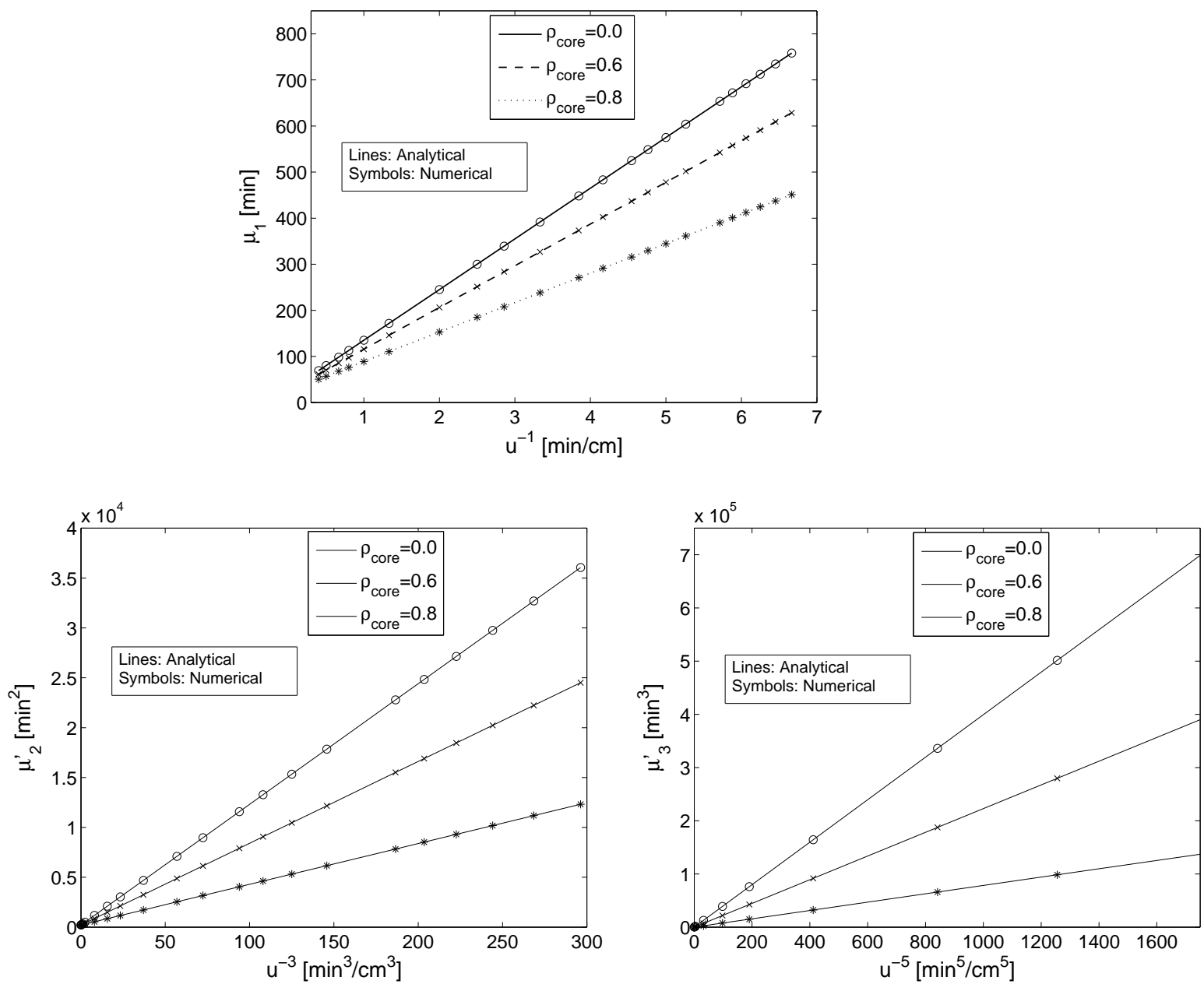

Figure 5: Moments of GRM for core-shell particles using Dankwerts BC [25]. Here, Here, $D_{\text {eff }}=2 \times$ $10^{-5} \mathrm{~cm}^{2} / \mathrm{min}, R_{p}=2 \times 10^{-4} \mathrm{~cm}, k_{\text {ext }}=5.0 \mathrm{~min}^{-1}$, and $D_{L}=0.1 \mathrm{~cm}^{2} / \mathrm{min}$. Other parameters are given in Table 1. 

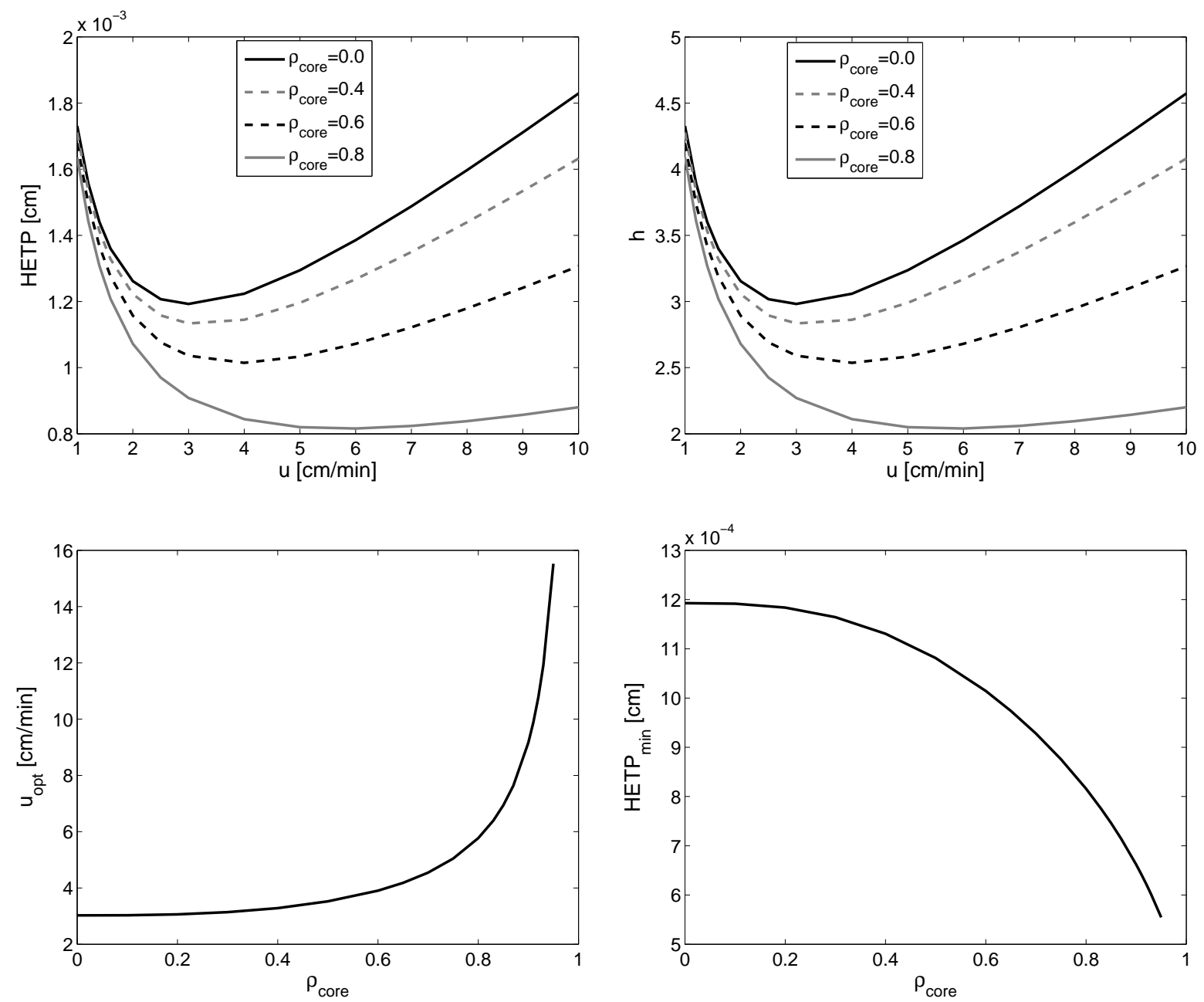

Figure 6: Plots of HETP over $u$ and $u_{\text {opt }}$ and HETP min $_{\text {over }} \rho_{\text {core }}$ using Dirichlet BC (c.f. Eqs. (30), (49) and (50)) [25]. Here, $\gamma_{1}=0.5, \gamma_{2}=1, D_{\text {mol }}=6 \times 10^{-4} \mathrm{~cm}^{2} / \mathrm{min}, D_{\text {eff }}=2 \times 10^{-5} \mathrm{~cm}^{2} / \mathrm{min}$, $R_{p}=2 \times 10^{-4} \mathrm{~cm}, k_{\mathrm{ext}}=5 \mathrm{~cm} / \mathrm{min}, D_{L}=0.1 \mathrm{~cm}^{2} / \mathrm{min}$, and other parameters are given in Table 1 . 

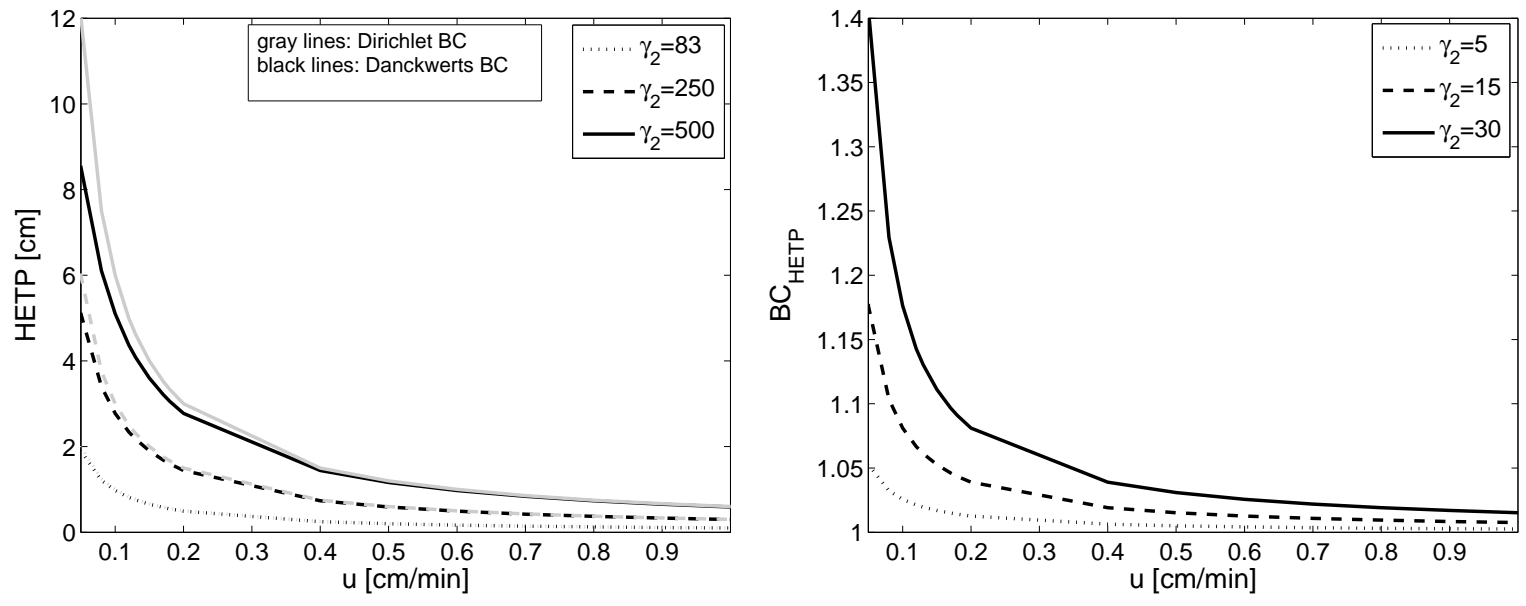

Figure 7: Plots of HETP and $\mathrm{BC}_{\text {HETP }}$ over $u$ for different values of $\gamma_{2}[25]$. Here, $\rho_{\text {core }}=0, \gamma_{1}=0.5$, $D_{\text {mol }}=6 \times 10^{-4} \mathrm{~cm}^{2} / \min , D_{\text {eff }}=2 \times 10^{-5} \mathrm{~cm}^{2} / \min , R_{p}=2 \times 10^{-4} \mathrm{~cm}, k_{\text {ext }}=5 \mathrm{~cm} / \mathrm{min}, D_{L}=$ $0.1 \mathrm{~cm}^{2} / \mathrm{min}$, and other parameters are given in Table 1 . 

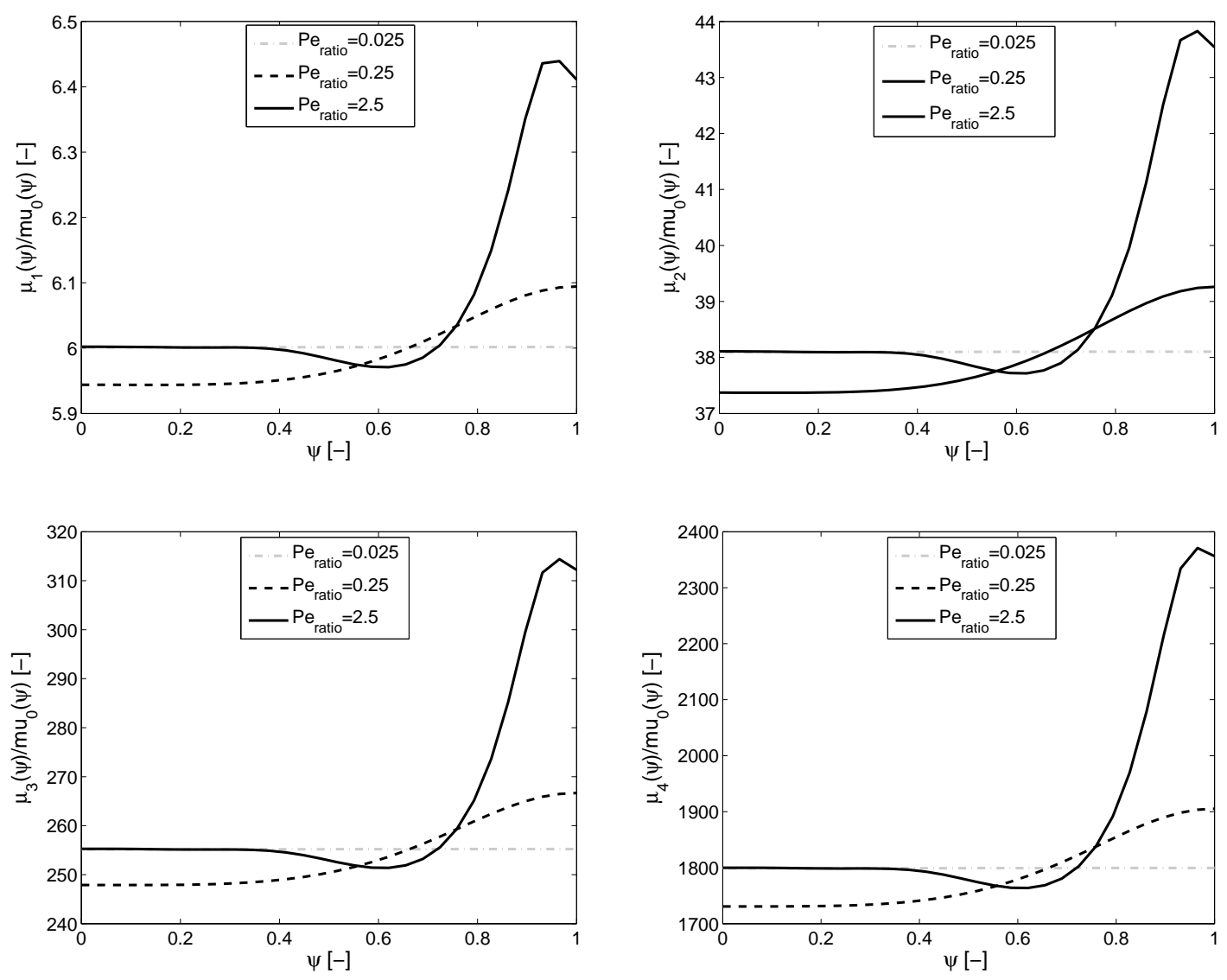

Figure 8: Inner zone injection: Plots of Local moments showing the effects of $P e_{\rho}[24]$. Here, $P e=60$, $\eta=2.0, B_{i}=50, R=0.2 \mathrm{~cm}, \tilde{\rho}=0.1414 \mathrm{~cm}, \epsilon=0.4 . \epsilon_{p}=0.333, \tau_{\mathrm{inj}}=1.0$, and $a=4$. Moreover, $P e_{\text {ratio }}=\frac{P e_{\rho}}{P e}$. 